\title{
Actualités internationales sur la radioprotection de l'environnement : état de l'art des connaissances, des méthodes et des pratiques
}

\author{
K. Beaugelin-Seiller^ et J. Garnier-Laplace \\ IRSN, PRP-ENV, SERIS, Cadarache, France.
}

Reçu le 29 avril 2015 - Accepté le 17 juin 2016

\begin{abstract}
Résumé - Les nouvelles normes de base européennes en radioprotection (2013/59/Euratom) introduisent des «critères environnementaux » pour la «protection de la santé humaine à long terme ». Cette nouveauté résulte des travaux en cours et du positionnement récent des organisations et instances internationales en charge de la radioprotection et de sa mise en œuvre, quant à la protection de la faune et de la flore vis-à-vis de l'exposition aux rayonnements ionisants. Sur la base d'un état de l'art international afférent, depuis le contexte réglementaire actuel jusqu'aux approches existantes, la méthode ERICA a été identifiée comme parfaitement cohérente avec l'approche de la CIPR et des plus opérationnelles en raison de sa richesse, de sa flexibilité et de sa traçabilité. Cette méthode graduée a été ainsi employée dans la majorité des études d'impact publiées depuis 5 ans, qui écartent généralement dès l'étape dite de «screening » le risque radiologique pour la faune et la flore, excepté pour certaines zones parmi les plus impactées par des contaminations radioactives (environs de Tchernobyl, mines d'uranium d'Asie Centrale,...). Cette mise à jour des connaissances dans le domaine au niveau de la littérature internationale et le retour d'expérience fondé sur l'analyse de dossiers d'exploitants ont conduit à 10 recommandations de l'IRSN sur la radioprotection de l'environnement et sa prise en compte.
\end{abstract}

\begin{abstract}
International news about radiation protection of the environment. The new European Basic Safety Standards in Radiation Protection (2013/59/Euratom) introduce "environmental criteria" to protect human health in the long term. This innovation results from work in progress and from the recent positioning of international organizations and authorities in charge of radiation protection and its implementation, regarding the protection of wildlife against ionizing radiation. On the basis of the international state of the art, from the current regulatory context to existing approaches, the ERICA method has been identified as fully consistent with the ICRP approach and the most operational at the moment, due to its richness, flexibility and traceability. Most of the radiological risk assessments for wildlife published in the 5 last years used part or all of the ERICA approach, generally screening the radiological risk for wildlife from the first stage of the assessment, except for a few areas among those most impacted by radioactive contamination (Chernobyl area, uranium mining sites in Central Asia, etc.). This update of the knowledge in the field at the international level and the feedback on the analysis of regulatory files presented by nuclear operators led to 10 recommendations from the IRSN on the radiation protection of the environment and its implementation.
\end{abstract}

Keywords: Radioprotection / environment / regulation / international / France

\section{Contexte}

La révision en 2013 de la Directive Européenne fixant les normes de bases en radioprotection (2013/59/Euratom; Conseil de l'Union Européenne, 2014) a introduit dans l'objectif de la protection à long terme de la santé humaine, la nécessité de prendre en considération $« \ldots$ des critères

* karine.beaugelin@irsn.fr environnementaux fondés sur des données scientifiques reconnues... ». Cette nouveauté résulte des travaux en cours et du positionnement récent des organisations et instances internationales en charge de la radioprotection et de sa mise en œuvre, en ce qui concerne la protection de la faune et de la flore visà-vis de l'exposition aux rayonnements ionisants.

Dans ce cadre, l'IRSN a produit à la demande de l'ASN un état de l'art à l'échelle internationale sur le thème de la radioprotection de l'environnement (IRSN, 2014). En s'appuyant 
notamment sur ces bases et sur son propre retour d'expérience, l'institut a émis une dizaine de recommandations sur les méthodologies et connaissances associées utilisées ou utilisables par les parties prenantes (exploitants, pouvoirs publics, associations) pour évaluer le risque pour la faune et la flore du fait de leur exposition aux rayonnements ionisants (IRSN, 2014). Le contexte d'application de ces recommandations couvre les situations d'exposition planifiée (e.g., fonctionnement normal des installations nucléaires), existantes (e.g., marquage ou pollution résultant d'un incident ou d'un accident) ainsi que les situations d'urgence et la phase post-accidentelle. Ce document, dont le contenu est résumé ici, a également servi de base pour nourrir la réflexion du groupe permanent en Radioprotection et en Environnement (GPRADE) sollicité sur ce thème par l'ASN.

\section{Aspects réglementaires actuels aux niveaux international et national}

En 2006, l'AIEA a introduit un septième principe fondamental de sûreté (IAEA, 2006), dédié à la protection des personnes et de l'environnement contre les rayonnements ionisants, pour les générations présentes et futures. Ce principe a ensuite été décliné dans la révision des normes de base internationales de radioprotection (IAEA, 2014a), en accord avec les dernières recommandations de la CIPR (ICRP, 2007) qui soulignent la nécessité d'un système de radioprotection de l'environnement en tant que tel et en complément du système de radioprotection de l'Homme. Par voie de conséquence, la Directive 2013/59/Euratom a intégré ces considérations, assurant ainsi la cohérence du cadre réglementaire européen avec les normes internationales.

En France, la protection de l'environnement fait partie intégrante du code de l'environnement, notamment au regard de l'étude d'impact environnemental. Il n'y a cependant à ce jour aucune mention explicite de la protection de l'environnement vis-à-vis des rayonnements ionisants en tant que telle dans la réglementation. Certains exploitants nucléaires ont cependant intégré une démonstration dans leurs études d'impact, d'une part par cohérence avec les attendus réglementaires en la matière pour les substances chimiques, d'autre part en raison de son exigence dans d'autres pays, notamment européens. L'exemple d'un exploitant présentant la méthodologie mise en œuvre et le retour d'expérience de cette intégration au sein de l'étude d'impact fait l'objet d'un article spécifique dans ce même numéro (Boyer et al., 2016).

Quelques nations (Canada, Finlande, Suède, Royaume-Uni et USA) exigent déjà réglementairement une démonstration explicite de l'absence de risque ou d'impact radiologique pour tout ou partie des écosystèmes exposés. Si les critères de protection et leurs valeurs numériques sont parfois donnés par le législateur, ils ne sont jamais considérés comme des normes au sens strict, qui impliqueraient une action en cas de dépassement. Ces critères constituent alors uniquement une information dans l'évaluation et la gestion du risque.

\section{3 État de l'art}

\subsection{Quelques repères temporels}

Au cours des 20 dernières années, les discussions sont allées crescendo concernant la nécessité de considérer faune et flore en termes de radioprotection, et non plus seulement comme vecteurs de contamination vers l'homme. Les années 90 furent une période charnière dans ce domaine : publications des premières valeurs de référence quant aux effets potentiellement induits (IAEA, 1992; UNSCEAR, 1996) ; signature de la convention OSPAR ${ }^{1}$, premier traité international dans lequel les substances radioactives sont explicitement citées au regard de la protection d'un milieu... Disposant d'une part des éléments issus de la radioprotection de l'homme, d'autre part des développements relatifs à l'évaluation du risque chimique pour les écosystèmes, tout était prêt à la fin du $\mathrm{XX}^{\mathrm{ème}}$ siècle pour élaborer une approche dédiée aux radionucléides et à l'environnement cohérente avec l'existant. Le pas suivant a été franchi en Europe avec la série de programmes scientifiques dédiés financés par la Communauté Européenne (2000-2008). FASSET (Framework for Assessment of Environmental Impact ; Larsson, 2004), ERICA (Environmental Risk from Ionising Contaminants : Assessment and Management; Beresford et al., 2007), PROTECT (Protection of the Environment from Ionising Radiation in a Regulatory Context; Howard et al., 2010) et STAR (STrategy for Allied Radioecology; Alonzo et al., 2016) ont ainsi contribué à l' accroissement des connaissances dans ce domaine et au final à l'émergence d'une approche intégrée et des outil et bases de données associés dédiés à l'évaluation et à la gestion du risque radiologique pour les écosystèmes.

\section{2 Éléments de base}

La méthodologie d'évaluation du risque, aujourd'hui reconnue applicable aux substances chimiques comme aux radionucléides, repose sur 3 composantes clés (analyse des expositions, analyse des effets et caractérisation du risque) articulées au sein d'une approche graduée qui comprend jusqu'à trois niveaux d'évaluation. L'enchainement de ces derniers répond au besoin de gagner en représentativité et de réduire les incertitudes associées au risque estimé : (1) le dépistage (ou screening), approche conservative faisant appel à des données génériques et des hypothèses majorantes, (2) l'évaluation générique, reposant sur la mise en œuvre de données génériques et hypothèses moins conservatives (e.g., valeurs moyennes) et (3) l'évaluation site-spécifique, nécessitant des informations locales.

Quel que soit le niveau d'évaluation, l'analyse des expositions a pour objectif de quantifier, pour chacune des voies d'exposition des organismes à protéger, les niveaux auxquels ils sont exposés. L'analyse des effets, fondée sur la caractérisation des relations entre exposition et effets écotoxiques,

1 Texte résultant de la fusion des conventions d'Olso et Paris, par lequel quinze gouvernements et l'Union Européenne coopèrent pour protéger l'environnement marin de l'Atlantique du Nord-Est (http:// www.ospar.org/). 
a pour objectif de déterminer des valeurs de référence adéquates pour l'évaluation (par exemple, valeurs prédites sans effet). La caractérisation du risque combine les résultats des 2 phases d'analyse afin d'identifier (et quantifier le cas échéant) l'existence d'un risque pour la faune et la flore.

Caractériser l'exposition de la faune et de la flore aux substances radioactives nécessite des facteurs de concentrations (FC), quantifiant les transferts des radionucléides aux organismes à partir de diverses voies d'exposition, et des coefficients de dose. La méconnaissance des FC est la source principale d'incertitude de cette phase.

En matière d'effets des rayonnements ionisants chez les espèces non humaines, la très grande majorité des connaissances est répertoriée au sein de la base de données FREDERICA (Copplestone et al., 2008), dont une méta-analyse permet de dériver par l'établissement de relations dose (débit de dose)intensité d'effet des valeurs de référence servant à caractériser le risque associé au niveau d'exposition estimé pour la faune et la flore.

\section{Approches existantes}

\subsection{L'approche de la CIPR}

Ses dernières recommandations pointant le besoin d'un système de radioprotection pour l'environnement, la CIPR s'attache depuis 2003 à formaliser l'approche qu'elle propose, en la documentant progressivement (ICRP, 2008, 2009, 2014).

Reprenant le concept ayant fait ses preuves en matière de radioprotection de l'homme (ICRP, 2002), la Commission fonde son système de radioprotection des autres espèces vivantes sur des animaux et plantes de référence (Reference Animals and Plants - RAPs). Ces entités hypothétiques sont nécessaires aux calculs dosimétriques. Elles permettent également de compiler les connaissances et les relations dose-effets biologiques pour les espèces taxonomiquement proches. Dans le cadre d'une évaluation d'impact spécifique, la notion de RAP est mise en correspondance avec un organisme représentatif (RO), introduit par analogie avec la personne représentative définie en radioprotection de l'homme.

Au principe de limitation de dose, jugé inapproprié pour l'environnement, est substitué le concept de DCRL (Derived Consideration Reference Levels). La limite de dose (dose totale maximale qu'un individu peut recevoir du fait de son exposition planifiée à une ou des sources réglementées, hors exposition médicale) correspond en effet à un critère individuel réglementaire propre aux situations d'exposition planifiée. Le DCRL est défini pour chaque RAP par la gamme de débits de dose (couvrant un ordre de grandeur) au sein de laquelle des effets délétères sont susceptibles d'apparaître pour des individus du même type que le RAP.

Cette approche a été adoptée par l'AIEA (IAEA, 2014b) pour être déclinée dans les guides internationaux en sûreté et radioprotection, dont celui en cours d'élaboration pour les situations d'expositions planifiées (IAEA, en préparation) et celui relatif au dumping en mer en support de la convention de Londres (IAEA, 2015). Un troisième document est en préparation sous l'égide d'OSPAR pour la protection de l'environnement marin de l'Atlantique Nord-Est.
Des réserves ont été émises sur l'approche de la CIPR, notamment en raison du caractère indirect de la protection qu'elle offre aux populations et aux communautés au sein des écosystèmes, du fait de l'incertitude associée à la nécessaire extrapolation à des niveaux d'organisation écologique supérieure d'effets écotoxiques observés à l'échelle de l'individu. Le Programme des Nations Unies pour l'Environnement (PNUE) souhaite également voir élargir les définitions des termes « environnement» et «protection de l'environnement» vers une perspective plus vaste au-delà des seules considérations d'effets radiologiques aux plantes et aux animaux, c'est-à-dire incluant aussi le maintien de la qualité de l'air, des sols et des eaux ainsi que des considérations de durabilité (IAEA, 2014b).

L'approche dite «écosystèmes » soutenue par l'UIR (2012) rejoint cette position. Elle a pour objectif l'évaluation directe des risques à un niveau d'organisation biologique supérieur, celui des populations d'espèces et des écosystèmes (Bradshaw et al., 2014). Destinée à protéger le vivant dans son ensemble (conservation de tous les milieux, toutes les espèces et de leur diversité génétique et valorisation des services écosystémiques), cette approche est complémentaire de celle de la CIPR.

\subsection{L'approche ERICA}

L'approche ERICA, la plus utilisée en Europe, intègre les aspects d'évaluation, de gestion et de communication du risque. Cette approche intégrée et graduée propose un dépistage, suivi si besoin d'une étape générique raffinant l'analyse de l'exposition des écosystèmes. Ces deux étapes font appel à un débit de dose sans effet attendu (PNEDR - Predicted NoEffect Dose Rate; Garnier-Laplace et al., 2006), intervenant dans le calcul déterministe d'un quotient de risque. L'étape ultime de l'approche propose d'utiliser des données site spécifiques et des méthodes probabilistes pour caractériser le risque. Elle est donc à la fois calculatoire et fondée sur des données de surveillance (concentrations de radionucléides dans les milieux et les espèces, suivis écologiques...).

\subsection{Compatibilité des approches}

Pour la démonstration du niveau de protection de la faune et de la flore lors de l'évaluation du risque et sa gestion, les approches existantes en matière de radioprotection de l'environnement (Copplestone et al., 2001 ; US-DOE, 2002 ; Environment Canada, 2006; Beresford et al., 2007; ICRP, 2008, $2009,2014)$ appliquent toutes le principe de gradation, plus ou moins explicitement, chacune des étapes comportant les 3 composantes clés précédemment décrites ( $c f$. Sect. 3.2).

La notion d'organismes de référence est également partagée, en raison du consensus selon lequel la diversité de la vie sauvage nécessite de faire appel à un jeu limité d'organismes modèles, support de l'harmonisation des modèles et des données associées utilisés, a minima lors du dépistage d'un risque radiologique environnemental. La nature de ces organismes et leur nombre varient d'une approche à l'autre.

Concernant les points de comparaison pour évaluer les effets potentiels de l'exposition aux rayonnements ionisants, 
la CIPR s'est singularisée en retenant une gamme de valeurs. Les autres approches s'appuient sur un critère numérique unique (type PNEDR). Il est à noter que la robustesse de l'évaluation repose avant tout sur ces valeurs, conférant ainsi une importance haute à la qualité de leur établissement et à sa traçabilité.

La comparaison des atouts et des faiblesses des principales approches recensées met en exergue la richesse et la cohérence de l'ensemble ERICA en termes d'organismes de référence, de radionucléides et de valeurs de référence. Sa flexibilité, sa convivialité et la traçabilité de ses composantes jouent également largement en sa faveur. Un point majeur est que l'approche ERICA intègre l'approche de la CIPR, tout en présentant un caractère plus opérationnel pour les applications à des situations d'expositions environnementales variées.

\section{Retour d'expérience international}

Les travaux et études incluant au moins en partie une évaluation de risque radiologique pour les écosystèmes se sont multipliés parallèlement à l'insertion progressive de la thématique dans le domaine réglementaire. Projets de recherche européens (MARINA II - Radiological exposure of the European Community from radioactivity in North European marine waters ; EPIC - Environmental protection from ionising contaminants in the Arctic), forums internationaux (BIOPROTA), programmes AIEA (EMRAS - Environmental Modelling for Radiation Safety - I \& II, MODARIA - Modelling and Data for Radiological Impact Assessments) ou études à la demande de l'association nucléaire mondiale (World Nuclear Association), tous ont considéré la question du risque écologie lié à la présence de radionucléides dans l'environnement, par une méthode ou une autre. Dans ce contexte, la reconnaissance internationale de la valeur de l'approche ERICA se traduit par le nombre d'évaluations réalisées sur cette base ces dernières années. Ainsi, sur la trentaine de publications relatives au risque radiologique pour l'environnement parues depuis 2010 dans le monde, plus de la moitié fait référence pour tout ou partie à cette approche.

De façon cohérente, quelle que soit l'approche adoptée, les auteurs des travaux recensés s'accordent pour conclure généralement à l'absence de risque significatif pour les écosystèmes exposés aux rayonnements ionisants en situation d'exposition chronique liée au fonctionnement normal des installations. Quelques situations réelles particulières conduisent cependant à des conclusions opposées. Ainsi, les estimations de débit de dose dans la zone d'exclusion de Tchernobyl, au voisinage du réacteur accidenté, excèdent régulièrement les critères proposés au plan international. Les conclusions des évaluations menées sur les mines d'uranium d'Asie centrale et des états voisins sont similaires, comme celles portant sur les cours d'eau d'ex-URSS impactés par des contaminations radioactives. Des simulations d'accident, notamment sur sous-marin nucléaire, laissent également présager d'un risque radiologique pour des mers peu profondes, dites sensibles.

Enfin, il est à noter que, dès l'année de sa survenue, les conséquences écologiques de l'accident de Fukushima ont été l'une des facettes des travaux entrepris au plan international, notamment dans le cadre de l'UNSCEAR (2014).

\section{Conclusions}

L'introduction dans la réglementation de concepts relatifs à la radioprotection de la faune et de la flore a rapidement suivi les développements méthodologiques entrepris par différentes instances, soulignant la reconnaissance d'un besoin en la matière. Tirant partie de l'existant, tant sur le plan de la radioprotection de l'homme que sur celui de l'évaluation du risque chimique pour l'environnement, une voie consensuelle s'est dégagée. Elle permet en l'état des connaissances d'évaluer l'impact radiologique d'une exposition chronique de la faune et de la flore, en assurant la cohérence des études d'impact sanitaire et écologique pour des substances stables et radioactives.

Les approches opérationnelles existantes ont des limites, mais constituent un outil de démonstration qui semble aujourd'hui indispensable et qui peut être appliqué aux situations d'exposition existante et planifiée. Ces méthodes ne sont cependant pas figées, et évoluent déjà avec l'acquisition de connaissances nouvelles répondant à leur limitation. Par ailleurs, la réflexion doit se poursuivre quant à leur mise en œuvre pour des situations d'urgence ou en phase post-accidentelle.

En France, il serait cohérent avec l'actuel code de l'environnement d'inclure aujourd'hui dans le cadre de tout projet impactant potentiellement l'environnement, par exemple au sein de l'étude d'impact environnemental, la démonstration systématique et proportionnée aux enjeux du niveau de protection radiologique de la faune et de la flore.

\section{Références}

Alonzo F., Hertel-Aas T., Réal A., Lance E., Garcia-Sanchez L., Bradshaw C., Vives i Batlle J., Oughton D.H., Garnier-Laplace J. (2016) Population modelling to compare chronic external radiotoxicity between individual and population endpoints in four taxonomic groups, J. Environ. Radioact. 152, 46-59.

Beresford N., Brown J., Copplestone D., Garnier-Laplace J., Howard B.J., Larsson C.-M., Oughton O., Pröhl G., Zinger I. (eds.) (2007) D-ERICA : An integrated approach to the assessment and management of environmental risks from ionising radiation. Description of purpose, methodology and application. Contract Number : FI6R-CT-2003-508847.

Boyer C., Gontier G., Hémidy P.Y. (2016) Radioprotection de l'environnement : méthodologie et retour d'expérience d'EDF. Radioprotection 51 (4), 237-244.

Bradshaw C., Kapustka L., Barnthouse L., Brown J., Ciffroy P., Forbes V., Geras'kin S., Kautsky U., Bréchignac F. (2014) Using an ecosystem approach to complement protection schemes based on organism-level endpoints, J. Environ. Radioact. 136, 98-104.

Conseil de l'Union Européenne (2014) Directive 2013/59/Euratom du Conseil de l'Union Européenne du 5 décembre 2013 fixant les normes de base relatives à la protection sanitaire contre les dangers résultant de l'exposition aux rayonnements ionisants et abrogeant les directives 89/618/Euratom, 90/641/Euratom, 96/29/Euratom, 97/43/Euratom et 2003/122/Euratom. Publié le 17 janvier 2014 au JOUE.

Copplestone D., Bielby S., Jones S.R., Patton D., Daniel D., Gize I. (2001) Impact Assessment of Ionising Radiation on Wildlife. Environment Agency, Bristol. 
Copplestone D., Hingston J., Real A. (2008) The development and purpose of the FREDERICA radiation effects database, $J$. Environ. Radioact. 99 (9), 1456-1463.

Environment Canada (2006) Liste des substances d'intérêt prioritaire - Rapport d'évaluation - Rejets de radionucléides des installations nucléaires (effets sur les espèces autres que l'être humain). Gouvernement du Canada, Québec.

Howard B.J., Beresford N.A., Andersson P., Brown J.E., Copplestone D., Beaugelin-Seiller K., Garnier-Laplace J., Howe P.D., Oughton D., Whitehouse P. (2010) Protection of the environment from ionising radiation in a regulatory context-an overview of the PROTECT coordinated action project, J. Radiol. Prot. 30 (2), 195-214.

Garnier-Laplace J., Della-Vedova C., Gilbin R., Copplestone D., Hingston J., Ciffroy P. (2006) First derivation of predicted-noeffect values for freshwater and terrestrial ecosystems exposed to radioactive substances, Environ. Sci. Tech. 40, 6498-6505.

IAEA (1992) Effects of ionizing radiation on plants and animals at levels implied by current protection standards. IAEA Technical report $n^{\circ} 332$.

IAEA (2006) Fundamental Safety Principles. IAEA Safety Standards for protecting people and the environment. Safety Fundamentals SF-1. International Atomic Energy Agency, Vienna, Austria.

IAEA (2014a) Radiation Protection and Safety of Radiation Sources : International Basic Safety Standards. General Safety Requirements Part 3 - No GSR Part 3. International Atomic Energy Agency, Vienna, Austria.

IAEA (2014b) Summary notes from the Technical Meeting of the Coordination Group on Radiation Protection of the Environment : input to Safety Standards taking into account the BSS and relevant ICRP/international recommendations. IAEA, Vienna, 2-3 July 2013, April 2014.

IAEA (2015) Determining the Suitability of Materials for Disposal at Sea under the London Convention 1972 and London Protocol 1996 : A Radiological Assessment Procedure. IAEA TECDOC 1759, International Atomic Energy Agency, Vienna, Austria.
IAEA (en préparation) Radiological Environmental Impact Assessment for Facilities and Activities, Draft Safety Standards DS427, International Atomic Energy Agency, Vienna, Austria.

ICRP (2002) Basic Anatomical and Physiological Data for Use in Radiological Protection Reference Values. ICRP Publication 89, Ann. ICRP 32 (3-4).

ICRP (2007) The 2007 Recommendations of the International Commission on Radiological Protection. ICRP Publication 103, Ann. ICRP 37 (2-4).

ICRP (2008) Environmental Protection - the Concept and Use of Reference Animals and Plants, ICRP Publication 108, Ann. ICRP 38 (4-6).

ICRP (2009) Environmental Protection : Transfer Parameters for Reference Animals and Plants, ICRP Publication 114, Ann. ICRP 39 (6).

ICRP (2014) Protection of the Environment Under Different Exposure Situations, ICRP Publication 124, Ann. ICRP 43 (1).

IRSN (2014a) Radioprotection de l'environnement - état de l'art et recommandations de l'IRSN. IRSN, fiche technique FT/AV/PRPENV/2014-019 support de l'avis IRSN/2014-000362.

IRSN (2014b). Avis IRSN/2014-000362 du 17 octobre 2014, http:// www.irsn.fr/FR/expertise/avis/Pages/Avis-aux-autorites.aspx\#. VvFlM6Nwa70.

Larsson C.-M. (2004) The FASSET Framework for assessment of environmental impact of ionising radiation in European ecosystems - an overview, Radiol. Prot. 24, A1-A12.

UNSCEAR (1996) Source and effects of ionizing radiation. Report to the General Assembly with Scientific Annex. United Nations, NY, USA.

UNSCEAR (2014) Assessment of the impact of the 2011 nuclear accident in Japan (Scientific Annex A to the UNSCEAR 2013 Report to the UN General Assembly).

US-DOE (2002) A graded approach for evaluating radiation doses to aquatic and terrestrial biota. DOE Standard DOE-STD-11532002, 3 modules.

Cite this article as: K. Beaugelin-Seiller, J. Garnier-Laplace. Actualités internationales sur la radioprotection de l'environnement : état de l'art des connaissances, des méthodes et des pratiques. Radioprotection 51(4), 231-235 (2016). 